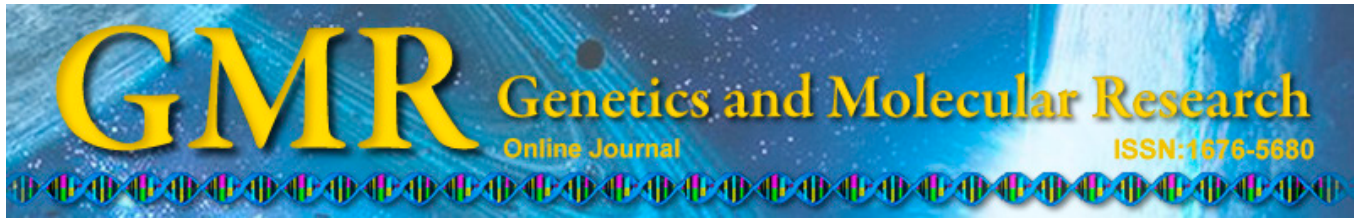

\title{
Postpartum pelvic floor function performance after two different modes of delivery
}

\author{
H. Li ${ }^{1,2}$, R.F. Wu ${ }^{1,2}$, F. Qi ${ }^{1,2}$, A.M. Xiao ${ }^{1,2}$, Z. Ma ${ }^{1,2}$, Y. Hu ${ }^{1,2}$, W.Y. Zhang ${ }^{1,2}$, \\ W. Li ${ }^{1,2}$ and Z.C. Wang ${ }^{1,2}$ \\ ${ }^{1}$ Department of Gynaecology, The Shenzhen Hospital, Peking University, \\ Senzhen, China \\ ${ }^{2}$ Shenzhen Key Laboratory of Gynecological Diagnostic Technology Research, \\ Shenzhen, Guangdong, China \\ Corresponding author: R.F. Wu \\ E-mail: RuiFengWUcn@163.com
}

Genet. Mol. Res. 14 (2): 2994-3001 (2015)

Received March 17, 2014

Accepted July 31, 2014

Published April 10, 2015

DOI http://dx.doi.org/10.4238/2015.April.10.9

\begin{abstract}
This study investigated the incidences of urinary incontinence and pelvic organ prolapse as well as pelvic floor muscle strength after cesarean section and vaginal delivery. From June 2010 to July 2011, 149 puerpera in Shenzhen Hospital, Peking University, were divided into the cesarean section group $(\mathrm{N}=66)$ and the vaginal delivery group $(\mathrm{N}=83)$. Postpartum urinary incontinence analysis, pelvic examination, and pelvic muscle contraction analysis using the PHENIX neuromuscular therapy instrument were performed to compare urinary incontinence, pelvic organ prolapse, and pelvic floor muscle condition between the 2 groups. The incidences of urinary incontinence in the cesarean and vaginal delivery groups were $9.09 \%$ $(6 / 66)$ and $16.87 \%(14 / 83)$, respectively $(\mathrm{P}>0.05)$; the incidences of pelvic organ prolapse were 53.03\% (35/66) and 86.75\% (72/83), respectively $(\mathrm{P}<0.05)$. There was no significant difference in pelvic muscle pressure or electrophysiological examination results between the 2 groups $(\mathrm{P}>0.05)$. Hence, cesarean section has a protective effect on early postpartum pelvic organ prolapse, but the delivery modes do
\end{abstract}


not differ significantly with respect to the incidence of postpartum urinary incontinence or pelvic muscle floor muscle strength.

Key words: Pelvic organ prolapse; Urinary incontinence; Pelvic floor muscle; Cesarean section; Vaginal delivery

\section{INTRODUCTION}

Female pelvic floor dysfunction is an increasingly important social and health issue. It includes pelvic organ prolapse (POP) and urinary incontinence, predominantly stress urinary incontinence. Pregnancy and birth are independent risk factors for female pelvic floor dysfunction (Strinić et al., 2007) that have been accepted by the general public. Therefore, women are increasingly opting for cesarean birth, with the aim of protecting pelvic floor function. However, there is insufficient evidence to confirm that cesarean section indeed has this advantage.

Before clinicians select cesarean section, it is necessary to fully weigh the pros and cons of the vaginal and cesarean section. Accordingly, this study compared the early postpartum pelvic floor function of women who underwent cesarean or vaginal delivery by analyzing the incidence of early maternal urinary incontinence within 1 year after delivery as well as gynecological pelvic examination and pelvic floor muscle strength measurement. Thus, this study aims to provide theoretical support for the clinical decision making of obstetricians.

\section{MATERIAL AND METHODS}

\section{Patients}

From June 2010 to July 2011, 149 puerparae from our hospital were enrolled; there were 83 cases of normal delivery and 66 cases of cesarean section. The inclusion criteria were puerparae who consented to inspection in our hospital 8-12 weeks after delivery, including primipara, singleton birth, age $<35$ years, $<20 \mathrm{~kg}$ weight gain during pregnancy, and neonate birth weight $<4 \mathrm{~kg}$. Puerparae with placenta previa, placental abruption, gestational diabetes, other pathological obstetrics and forceps, and a history of vacuum suction dystocia were excluded.

\section{Study methods}

The following analyses were performed 8-12 weeks after delivery.

\section{Urinary incontinence}

In accordance with the diagnostic criteria published by the International Continence 
Society (ICS), the women filled out the International Urinary Incontinence Questionnaire, the Short-Form Health Questionnaire (SF-36), and Pelvic Floor Dysfunction-Associated Diseases Questionnaire (Garcia-Burguillo and Aguila-Maturana, 2009).

\section{Pelvic examination}

All puerparae underwent conventional gynecological, urinary incontinence, and pelvic organ prolapse examinations in order to determine if they had urinary incontinence. Evaluated parameters included the inability to hold urine, unconscious urine leakage as well as the presence of pelvic organ prolapse symptoms such as conscious tenesmus and vulvar herniation; parameters were scored and indexed according to the International Standard of Urinary Incontinence and POP-Q.

\section{Pelvic floor muscle strength measurement}

For the electrophysiological examination, a muscle potential probe (Guangzhou Shanshan Company, China) was inserted into the vagina; the other end was connected to the PHENIX neuromuscular therapy instrument (Electronic Concept Lignon Innovation, France), which was used to detect muscle force changes of type I and II muscle fibers.

Muscle strength was classified as grade 0 if it reached $40 \%$ of maximum strength within $10 \mathrm{~s}$ and was highly persistent for $0 \mathrm{~s}$; it was classified as grades I, II, III, IV, and V if it was persistent for $1,2,3,4$, and $\geq 5 \mathrm{~s}$, respectively.

\section{Muscle strength of pelvic floor type II muscle fibers}

Muscle strength was classified according to the number of times each patient was able to rapidly contract and relax the pelvic floor muscles within $10 \mathrm{~s}$ to reach $70-90 \%$ of maximum muscle strength: $0,1,2,3,4$ and $\geq 5$ times was classified as grades 0 , I, II, III, IV, and $\mathrm{V}$, respectively.

The PHENIX neuromuscular therapy instrument was used to measure pressure (South et al., 2009). When the vagina is relaxed, pelvic floor muscle strength measured by the pressure probe is equivalent to the tension in the vagina, which reflects vaginal receptivity and basic tension. The difference between maximum systolic blood pressure and resting pressure is equivalent to the pelvic floor muscle contraction capacity.

Type I muscle fiber duration refers to the maximum pelvic floor muscle contraction duration after the contraction reaches $40-60 \%$ of maximum contraction within $10 \mathrm{~s}$. Type I muscle fiber sustained systolic pressure refers to the average of the maximum and minimum of type I muscle fiber contraction minus resting pressure, which is equivalent to the contraction intensity of type I pelvic floor muscle fibers. Type II muscle fiber contraction duration refers to the number of times a woman contracts pelvic floor muscle type II muscle fibers in $10 \mathrm{~s}$ (i.e., rapid contraction and the detected pelvic floor pressure is at least $70 \%$ of maximum contraction). Type II muscle fiber rapid systolic pressure refers to the maximum pressure achieved by the rapid contraction of type II muscle fibers minus 
maximum resting pressure, which is equivalent to the maximum contraction intensity of type II muscle fibers.

\section{Statistical analysis}

SPSS version 19.0 was used to analyze the data. Numerical and categorical data were analyzed by the $t$-test and the $\chi^{2}$ test, respectively. The level of significance was set at $\mathrm{P}<0.05$.

\section{RESULTS}

\section{General characteristics}

There were no statistically significant differences between groups with respect to gestational age, body mass index, or neonate body weight (Table 1).

Table 1. Comparison of gestational age, BMI, and neonate weight 8-12 weeks postpartum.

\begin{tabular}{lccc}
\hline & Labor age & BMI & Neonate weight $(\mathrm{kg})$ \\
\hline Vaginal delivery $(\mathrm{N}=83)$ & $28.88 \pm 2.10$ & $26.31 \pm 2.77$ & $3.19 \pm 0.38$ \\
Cesarean section $(\mathrm{N}=66)$ & $28.82 \pm 2.42$ & $25.49 \pm 2.55$ & $3.28 \pm 0.33$ \\
$t$ & -0.165 & -1.183 & 1.595 \\
$\mathrm{P}$ & $>0.05$ & $>0.05$ & $>0.05$ \\
\hline
\end{tabular}

$\mathrm{BMI}$, body mass index. Data are reported as means $\pm \mathrm{SD}$.

\section{Incidences of urinary incontinence and POP}

The incidence of urinary incontinence in the cesarean section group was 9.09\% (6/66), which was lower than that of $16.87 \%(14 / 83)$ for the normal delivery group, but the difference was not statistically significant $(\mathrm{P}>0.05)$. The incidence of POP in the cesarean section group was $53.03 \%(35 / 66)$, which was significantly lower than $86.75 \%(72 / 83)$ in the vaginal delivery group $(\mathrm{P}<0.05$; Table 2$)$.

Table 2. Incidence of urinary incontinence symptoms and POP between cesarean section and vaginal delivery.

\begin{tabular}{lcc}
\hline & Incidence of urinary incontinence $(\%)$ & Incidence of POP $(\%)$ \\
\hline Vaginal delivery $(\mathrm{N}=83)$ & $16.87(14 / 83)$ & $86.75(72 / 83)$ \\
Cesarean section $(\mathrm{N}=66)$ & $9.09(6 / 66)$ & $53.03(35 / 66)$ \\
$\chi^{2}$ & 1.19 & 20.65 \\
$\mathrm{P}$ & $>0.05$ & $<0.05$ \\
\hline
\end{tabular}

POP, pelvic organ prolapse.

\section{Pelvic floor muscle pressure}

Postpartum pelvic floor muscle pressure and electrophysiological examinations were performed using the PHENIX neuromuscular therapy instrument. However, there was no significant difference in pelvic floor muscle strength between groups (Table 3). 


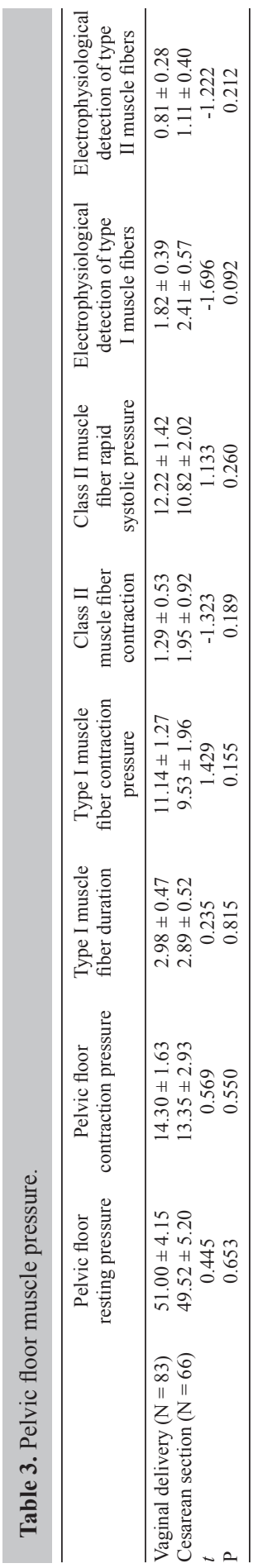




\section{DISCUSSION}

The main components of the pelvic support system include the pelvic floor muscles (i.e., levator ani), fasciae, and ligaments. Miedel et al. (2009) and Campeau et al. (2011) divide the vaginal support structure into 3 levels. The first level (i.e., top support) refers to the uterosacral ligament including the cardinal ligament complex, vertically supporting the uterus, and the upper one-third of the vagina; this provides the main support strength for the pelvic floor. The second level (i.e., horizontal support) refers to the linea alba formed by the pubocervical fascia attached to the tendinous arch and the midline of the rectovaginal fascia as well as the levator ani muscle, horizontally supporting the bladder, the upper two-thirds of the vagina, and rectum. The third level (i.e., distal support) refers to the distal pubocervical fascia and rectovaginal fascia extension and merges in the perineal body, supporting the distal urethra, which mutual coordinates and maintains the uterus, bladder, rectum, and pelvic viscera in their normal positions.

Numerous domestic and foreign epidemiological studies show that the factors related to female pelvic floor dysfunction include age, obesity, menopause, pregnancy and vaginal delivery, pelvic operation, and urinary tract infection (Nygaard et al., 2008). Among these factors, pregnancy and childbirth are most closely related to female pelvic floor dysfunction. In the process of pregnancy and childbirth, chronic fetal oppression of the pelvic floor musculature and innervation of the supporting tissue of the pelvic cavity can injure the pelvic floor muscles and cause urethral sphincter denervation damage; the fetus passing through the soft birth canal can also directly damage the pelvic floor, anal sphincter, and perineum (Payne, 2008; Zahariou et al., 2008; Shek et al., 2012).

The pelvic floor muscles comprise type I and II muscle fibers. Type I muscle fibers are slow-contraction fibers that maintain tension; they are long, durable, do not fatigue easily, and are characterized by tetanic contraction. They are the main component of the pelvic floor muscles. Meanwhile, type II muscle fibers are rapid-contraction muscle fibers that maintain reflexes and voluntary contractions; they are fast, transient, fatigue easily, and are characterized by phasic contraction. Clinical manifestations of decreased type I pelvic floor muscle strength include vaginal relaxation, recurrent urinary tract infections, uterine and other organ prolapse, and continuous postural urine leakage; meanwhile, clinical manifestations of decreased type II pelvic floor muscle strength include inability to contract the pelvic floor muscles and frequent urine leakage during body movements such as coughing, laughing, and general movement.

Whether cesarean section can reduce the incidence rate of postpartum urinary incontinence remains controversial (Wesnes et al., 2009; Twomey and Robinson, 2010). In Canada, Farrell et al. (2001) found that at 6 months postpartum, the incidence of urinary incontinence in women who underwent vaginal delivery was $26 \%$, including obvious clinical symptoms in $4 \%$ of patients. Meanwhile, the urinary incontinence rate in women who underwent cesarean section was $10 \%$. Cesarean section is reported to reduce the incidence of postpartum urinary incontinence by $5-10 \%$. Furthermore, this protective effect can be sustained up to the age of 50 ; thereafter, the prevalence of urinary incontinence is unaffected by the influence of delivery mode. Groutz et al. (2004) found that the incidence of urinary incontinence in women who underwent cesarean section and vaginal delivery was $10.3-12 \% 1$ year postpartum; in addition, the incidence of urinary incontinence in the cesarean section group was lower than that in the vaginal delivery group, but the difference was not statistically significant. In the present study, the incidence of postpartum urinary incontinence was higher in the vaginal delivery 
group (16.87\%) than the cesarean section group $(9.09 \%)$, which is consistent with the report of Groutz et al. (2004); however, the difference was not significant, suggesting cesarean section does not reduce the incidence of postpartum urinary incontinence. However, this finding may be due to the small study sample size. Therefore, larger studies are required to confirm this.

There are also many reports about postpartum POP (Doshi et al., 2010). The risk of pelvic organ prolapse in vaginal delivery is 1.2 times higher than that in cesarean section. Similarly, women who have experienced 2 vaginal deliveries have a risk of pelvic organ prolapse 8.4 times that of women who have undergone cesarean section. Furthermore, a study in Turkey shows that 4 or more vaginal deliveries increases the risk of organ prolapse risk 12 times. In the present study, the early postpartum incidence of POP in the cesarean section group was $53.03 \%$, which was significantly lower than that of the vaginal delivery group (86.75\%), indicating cesarean section has a protective effect on the pelvic floor support structure in the early postpartum period. However, whether this protective effect is temporary requires longer follow-up.

The forward shift of the celiac gravity axis during pregnancy places abdominal pressure and the pelvic viscera center of gravity on the pelvic floor muscles; this combined with the increased uterus weight causes the pelvic floor structure to undergo long-term continuous stretching and compression, gradually causing varying degrees of damage to the pelvic floor fasciae, muscles, and ligaments as well as the integral relaxation of the pelvic floor.

Luo et al. (2011) measured pelvic floor muscle strength 42 days postpartum in 400 women who received pelvic floor muscle strength treatment; they found no significant difference in muscle strength between cesarean section and vaginal delivery. The present study used the PHENIX neuromuscular therapy instrument for pressure and electrophysiological examinations of postpartum pelvic floor muscle strength. However, there was no significant difference in early postpartum pelvic floor muscle strength between the cesarean section and vaginal delivery groups (Table 3). This finding is consistent with the results of Luo et al. (2011), indicating that the effects of pregnancy and delivery on pelvic floor muscle strength mainly occur during pregnancy itself.

Therefore, the protective effect of cesarean section on early postpartum pelvic floor support structure is limited. However, it is also important to consider the maternal harm of cesarean section. First, cesarean section results in higher maternal mortality than vaginal delivery (Ben Brahim et al., 2008; Jastrow et al., 2010). Second, cesarean section may cause complications including thromboembolic complications, heart- and lung-related diseases, operative trauma, and puerperal infection (Adanu and McCarthy, 2007; Jabir and Smeet, 2009). Third, cesarean section has long-term impacts on reproductive health; during the second maternal pregnancy, the risks of uterine rupture, placenta implantation, and placenta previa increase significantly (Turner et al., 2009).

In summary, cesarean section has a protective effect on early postpartum POP, but its long-term impact requires further study. Whether pregnant women should undergo cesarean section requires additional consideration. In particular, the prevention of POP cannot be used as an indication for cesarean section. The present study also shows that the incidence of postpartum urinary incontinence and pelvic floor muscle strength do not differ significantly between different delivery modes; however, further research is required, because there were few patients in this study.

\section{REFERENCES}

Adanu RM and McCarthy MY (2007). Vaginal birth after cesarean delivery in the West African setting. Int. J. Gynaecol. Obstet. 98: 227-231. 
Ben Brahim F, Zeghal D, Mahjoub S, Ben Hmid R, et al. (2008). Prognosis of delivery from a scared uterus: 123 cases. Tunis. Med. 86: 987-991 [in French].

Campeau L, Gorbachinsky I, Badlani GH and Andersson KE (2011). Pelvic floor disorders: linking genetic risk factors to biochemical changes. BJU Int. 108: 1240-1247.

Doshi AM, Van Den Eeden SK, Morrill MY, Schembri M, et al. (2010). Women with diabetes: understanding urinary incontinence and help seeking behavior. J. Urol. 184: 1402-1407.

Farrell S, Allen V and Baskett T (2001). Partuition and urinary incontinence in primiparas. J. Obstet. Gynecol. 97: 350 356.

Garcia-Burguillo MP and Aguila-Maturana AM (2009). Energy-saving strategies in the treatment of fatigue in patients with multiple sclerosis. A pilot study. Rev. Neurol. 49: 181-185 [in Spanish].

Groutz A, Rimon E, Peled S, Gold R, et al. (2004). Caesarean section: does it really prevent the development of postpartum stress urinary incontinence? A prospective study of 363 women one year after their first delivery. J. Neurol. Urodyn. 23: 2-6.

Jabir M and Smeet RI (2009). Comparison of oral and vaginal misoprostol for cervical ripening before evacuation of first trimester missed miscarriage. Saudi Med. J. 30: 82-87.

Jastrow N, Chaillet N, Roberge S, Morency AM, et al. (2010). Sonographic lower uterine segment thickness and risk uterine scar defect: a systematic review. J. Obstet. Gynaecol. Can. 32: 321-327.

Luo Z, Xiao J, Zhang HY, et al. (2011). The analysis of different modes of delivery on early postpartum pelvic floor muscle damage. Chin. J. Modern Drug Appl. 5: 18-19.

Miedel A, Tegerstedt G, Mahle-Schmidt M, Nyrén O, et al. (2009). Nonobstetric risk factors for symptomatic pelvic organ prolapse. Obstet. Gynecol. 113: 1089-1097.

Nygaard I, Barber M, Burgio K, Kenton K, et al. (2008). Prevalence of symptomatic pelvic floor disorders in US women. JAMA 300: 1311-1316.

Payne CK (2008). Epidemiology, pathophysiology, and evaluation of urinary incontinence and overactive bladder. Urology 51: 3-10.

Shek KL, Kruger J and Dietz HP (2012). The effect of pregnancy on hiatal dimensions and urethral mobility: an observational study. Int. Urogynecol. J. 23: 1561-1567.

South MM, Stinnett SS, Sanders DB and Weidner AC (2009). Levator ani denervation and reinnervation 6 months after childbirth. Am. J. Obstet. Gynecol. 200: 519.e1-7.

Strinić T, Buković D, Roje D, Milić N, et al. (2007). Epidemiology of pelvic floor disorders between urban and rural female inhabitants. Coll. Antropol. 31: 483-487.

Turner CE, Young JM, Solomon MJ, Ludlow J, et al. (2009). Incidence and etiology of pelvic floor dysfunction and mode of delivery: an overview. Dis. Colon Rectum 52: 1186-1195.

Twomey F and Robinson K (2010). Pilot study of participating in a fatigue management programme for clients with multiple sclerosis. Disabil. Rehabil. 32: 791-800.

Wesnes SL, Hunskaar S, Bo K and Rortveit G (2009). The effect of urinary incontinence status during pregnancy and delivery mode on incontinence postpartum. A cohort study. BJOG 116: 700-707.

Zahariou AG, Karamouti MV and Papaioannou PD (2008). Pelvic floor muscle training improves sexual function of women with stress urinary incontinence. Int. Urogynecol. J. Pelvic Floor Dysfunct. 3: 401-406. 